\title{
Research Paper: \\ The Effect of Sucking Ice Bits on Nausea and Vomiting During Chemotherapy in Patients with Breast Cancer
}

\author{
Mohammad Haddadi ${ }^{1} \odot$, Javad Ganjloo ${ }^{1} \odot$, Hamid Reza Hashemifard ${ }^{2} \odot$, Yasser Tabarraie ${ }^{3} \odot$, Hamid Robat Sarpooshi $^{1} \odot$
}

1. Department of Nursing, School of Nursing and Midwifery, Sabzevar University of Medical Sciences, Sabzevar, Iran.

2. Training, Research, Medical Center of Vasei Hospital, Sabzevar, Iran.

3. Department of Epidemiology and Biostatistics, School of Public Health, Tehran University of Medical Sciences, Tehran, Iran.

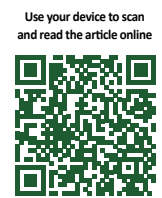

Cttation: Haddadi M, Ganjloo J, Hashemifard HR, Tabarraie Y, Robat Sarpooshi H. [The Impact of Sucking Bits of Ice on Nausea and Vomiting During Chemotherapy in Patients with Breast Cancer (Persian)]. Complementary Medicine Journal. 2019; 9(3):3804-3811. https://doi.org/10.32598/cmja.9.3.3804

https://doi.org/10.32598/cmja.9.3.3804

Key words: Ice, Nausea, Vomiting, Chemotherapy, Breast cancer

\section{ABSTRACT}

Objective Cancer disease and its treatment methods affect the quality of life of those suffering from breast cancer. Nausea and vomiting are frequent side-effects of chemotherapy. Nowadays, complementary therapies are used along with routine treatments to control this disease. This study aimed to evaluate the effect of sucking ice bits with plain water on nausea and vomiting during chemotherapy in patients with breast cancer.

Methods In this clinical trial, participants were 60 breast cancer patients undergoing chemotherapy. They were randomly divided into two groups of control and ice. The ice group received 30 bits of ice in $1 \mathrm{cc}$ during chemotherapy, while control group received 30-cc tap water. Nausea was evaluated based on visual analog scale and vomiting was estimated based on the number of cases. The data were analyzed in SPSS software.

Results The Mean \pm SD of nausea in ice group was $1.268 \pm 1.9$ and in the control group it was $1.787 \pm 2.9$ and this difference was statistically significant after intervention $(P=0.015)$. The mean of vomiting in ice group was $0.406 \pm 0.2$ and in the control group it was $0.449 \pm 0.266$, but this difference was not significant; i.e. the intervention had no significant effect on vomiting $(P=0.549)$.

Conclusion Sucking ice bits containing plain water had high effect on reducing nausea caused by chemotherapy but it had no effect on occurrence of vomiting.

\section{Extended Abstract}

\section{Introduction}

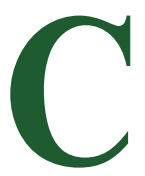

ancer is one of the most common diseases and problems in human societies. According to studies, breast cancer is the third most common cancer in the world and is the second leading cause of death among women after lung cancer. The treatment of breast cancer is complex and includes chemotherapy, radiotherapy, and surgery. Chemotherapy is one of the main, oldest, and most common cancer treatments that have the greatest impact on patients' quality of life following cancer, causing disruption to their physical, mental, social, and spiritual well-being.

Among these complications, nausea and vomiting are the most common side effects of these drugs experienced by

\section{* Corresponding Author:}

Hamid Robat Sarpooshi, MSc.

Address: Department of Nursing, School of Nursing and Midwifery, Medical Sciences of Sabzevar University, Sabzevar, Iran.

Tel: +98 (937) 0432422

E-mail: sarpooshi92@gmail.com 
about $40-80 \%$ of patients. These complications caused by chemotherapy has a devastating effect on patients' quality of life. These complications can be disruptive in many ways. The attempts to treat nausea and vomiting have not been effective and $60 \%$ of these patients still suffer from nausea and vomiting. On the other hand, various nausea and vomiting medications such as dexamethasone, metoclopramide, and ondansetron may lead to complications such as headache, dizziness, constipation, insomnia, and extrapyramidal effects. Therefore, nowadays, complementary therapies are used to treat this complication along with conventional treatments. This study aimed to investigate the effect of sucking sucking ice bits with plain water on nausea and vomiting during chemotherapy in patients with breast cancer.

\section{Materials and Methods}

This is a clinical trial conducted on 60 patients with breast cancer who referred to oncology ward of Omid Hospital in Mashhad, Iran from September to November 2015. They all were receiving adriamycin and cyclophosphamide for treatment of chemotherapy regimen. They were randomly divided into two control and intervention (ice) groups. Data collection tools included a demographic form (surveying age, educational level, marital status, disease grade), Visual Analogue Scale (VAS) for evaluating nausea, and a table for recording the number of vomiting cases completed by the patient, his/her companion or, if necessary, by the researcher.

The intervention was performed in both groups from 5 minutes before starting chemotherapy until the end of receiving chemotherapy medications. At first, both groups went under nausea and vomiting evaluation by using VAS. In the control group, in addition to routine treatment (granisetron and dexamethasone), patients received 30-cc tap water to consume $5 \mathrm{~min}$ before and during chemotherapy, and then their nausea and vomiting were checked in the end of chemotherapy. In the ice group, in addition to routine treatment (granisetron, dexamethasone), patients received 30 $1 \times 1$ ice bits containing 1-cc plain water to consume every $5 \mathrm{~min}$ (before and during chemotherapy), and in the end of chemotherapy, nausea and vomiting rates were recorded in both groups. In the end, the data were compared and analyzed in SPSS V. 16 software using t-test.

\section{Results}

The study groups were compared in terms of demographic characteristics using Chi-square and independent t-test whose results showed no significant difference between them. they were also homogenous in terms of nausea and vomiting rate before the intervention, but after the intervention, the mean of nausea in the ice group (1.28 \pm 1.9$)$ and the control group $(1.787 \pm 2.9)$ showed the significant difference between groups which indicates the effectivness of intervention $(\mathrm{P}<0.015)$; however, the obtained mean of vomiting in the ice group $(0.406 \pm 0.2)$ and control group $(0.449 \pm 0.226)$ indicated no significant difference between groups $(\mathrm{P}>0.549)$.

\section{Conclusion}

According to the results of this study, using ice containing plain water is recommended as a non-invasive, simple, and inexpensive, method with no side effects along with pharmacotherapy to reduce nausea in breast cancer patients. However, our findings on vomiting showed no effect of sucking ice containing plain water. Hence, further studies with larger sample sizes are recommended.

This study had some limitations. For example, due to the course of the disease and the condition of the patients with breast cancer, they felt a sense of belonging and ownership of their environment and medical staff; therefore, establishing communication with patients to carry out a research project was very difficult. It was resolved somehow by the collaboration of experienced chemotherapy personnel. Also, in this study, patients were not homogenized in terms of the number of chemotherapy sessions, which should be noted.

\section{Ethical Considerations}

\section{Compliance with ethical guidelines}

This study was extracted from a research proposal approved by the Research Ethics Committee of Sabzevar University of Medical Sciences (Code: IR.MEDSAB. REC.1394.53). It is also a registered clinical trial (code: IRCT20160606028293N2).

\section{Funding}

This study received financial support from the Sabzevar University of Medical Sciences.

\section{Authors' contributions}

Conceptualization, sampling and draft preparation by Mohammad Haddadi, Hamid Robat Sarpooshi and Javad Ganjloo; consulting for the identification and evaluation of patients by Hamid Reza Hashemifard; Data analysis by Yasser Tabarraie. 
Conflicts of interest

The authors declare no conflict of interest.

Acknowledgements

The authors would like to thank the patients and the emergency department staff of the Omid Hospital in Mashhad, Iran for their valuable cooperation and support. 


\title{
تأثير مكيدن يخ حاوى آب ساده بر تهوع و استفراغ حين شيمىدرمانى در مبتلايان به سرطان
}

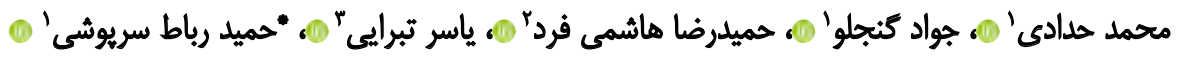 \\ ا. كروه يرستارى، دانشكده يرستارى و مامايى، دانشعاه علوم يزشكى سبزوار، سبزوار، ايران.

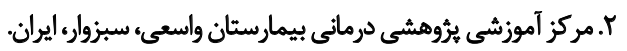 \\ r. كروه إيدميولوزى و آمار زيستى، دانشكده بهداشت، دائشكاه علوم يزشكى تهران، تهران، ايران.
}

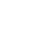

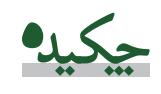

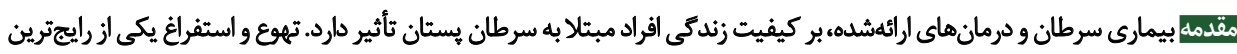

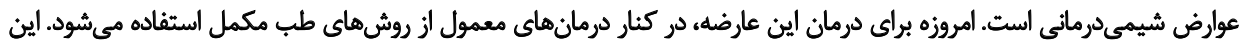

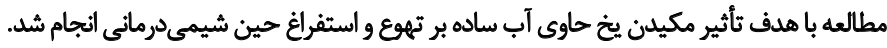

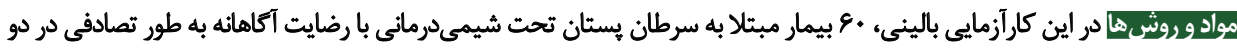

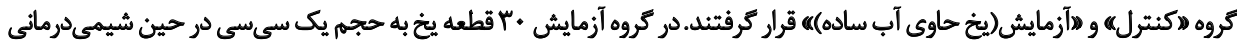

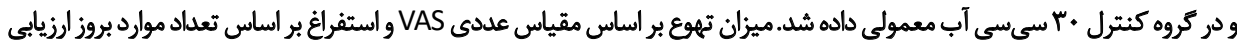

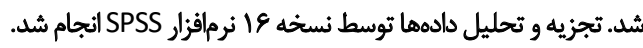

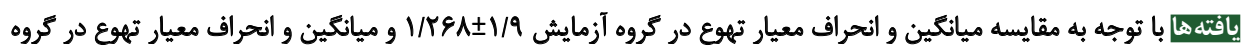

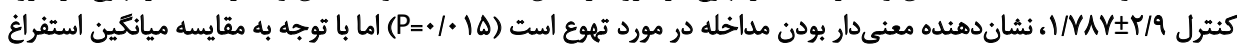

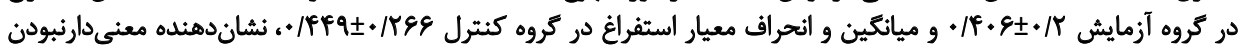

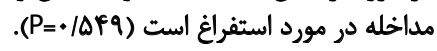

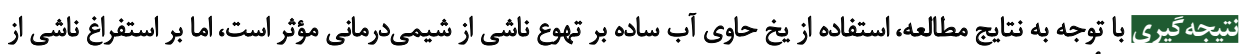
شيمى درمانى ثأثيرندارد.

اطلاعات مقاله:

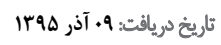

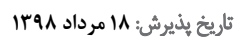

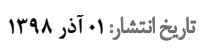

كليدوائهها: يخح، تهوع، استفراغا،

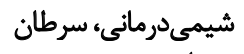
بستان
\end{abstract}

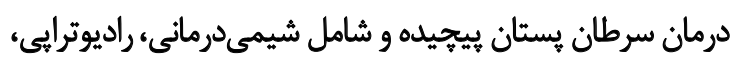

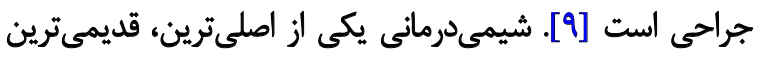

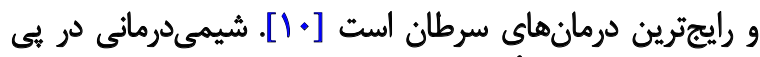

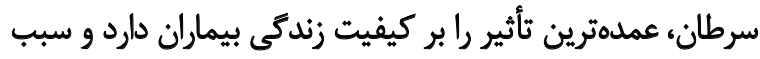

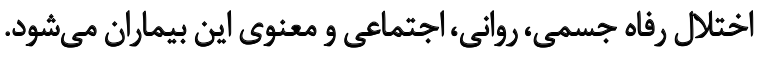

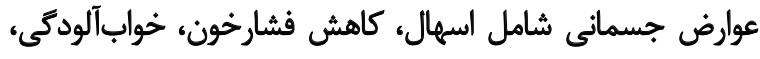

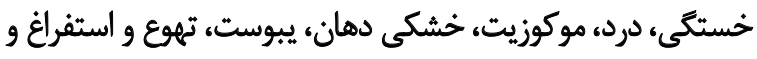

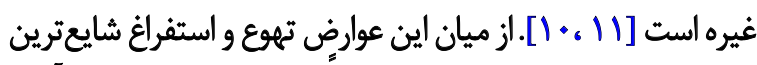

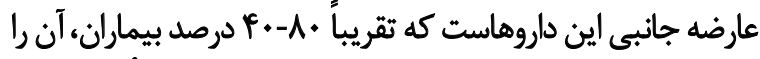

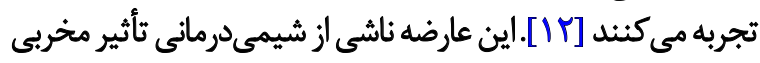

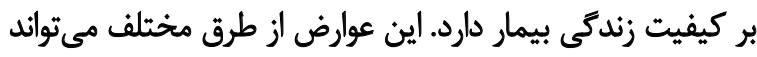

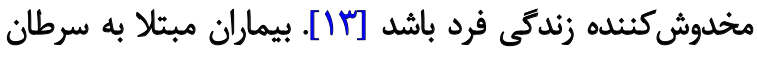

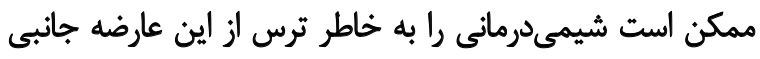

مقدمه

سرطان يكى از مهمثرين مشكلات و بيمارى شايع جوامع بشرى

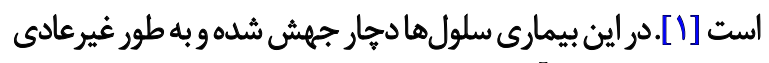

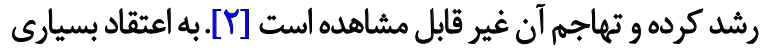

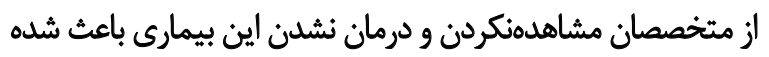

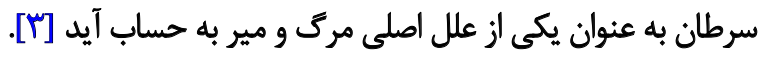

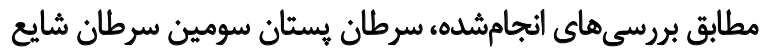

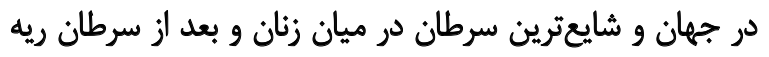

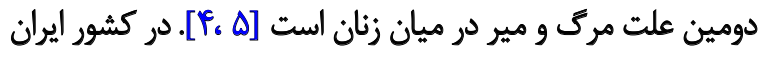

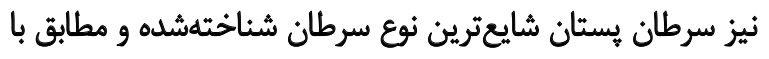

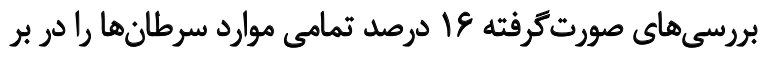

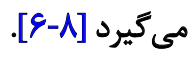

$$
\begin{aligned}
& \text { : تويسنده مسئول: }
\end{aligned}
$$

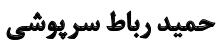

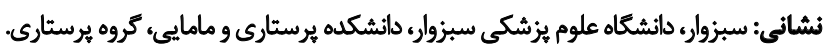

$$
\begin{aligned}
& \text { Tلفن: } \\
& \text { يست الكترونيكي: sarpooshi92@gmail.com }
\end{aligned}
$$


بررسى تهوع و جدول ثبت تعداد موارد استفراغ كه توسط بيمار يا

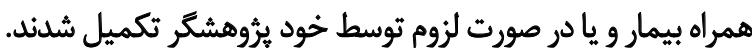

معيار ورود بيماران به مطالعه شامل رضايت كتبى جهت

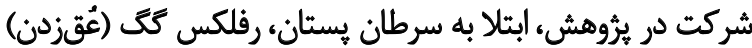

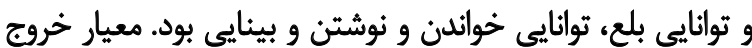

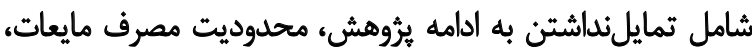

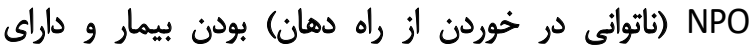

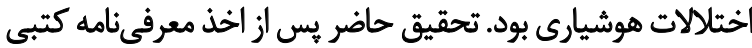

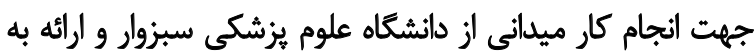

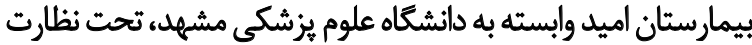

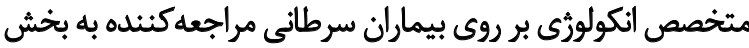

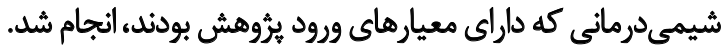
بيمار ان مراجعه كننده داراى سرطان يستان به روش آسان انتخاب

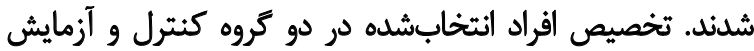

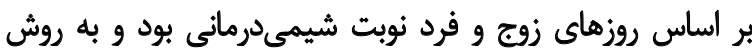

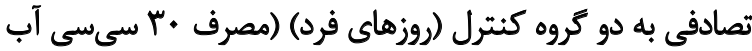

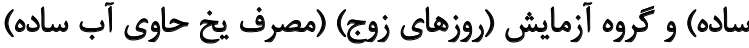

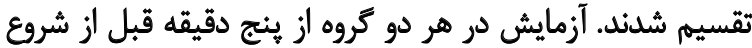

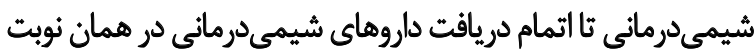

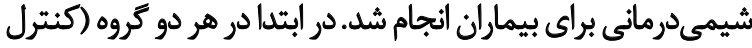

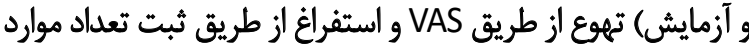

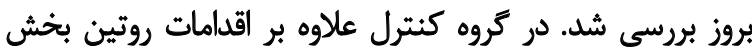

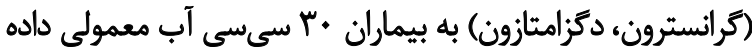

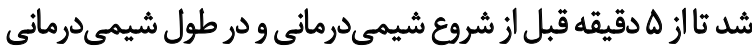

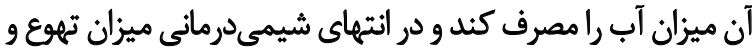

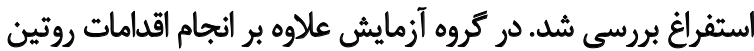

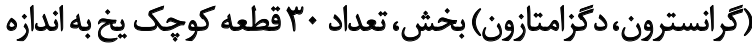

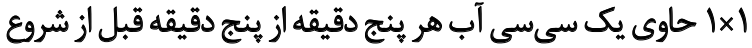

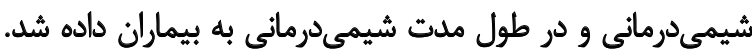

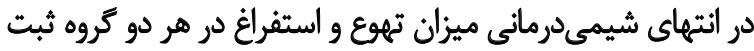

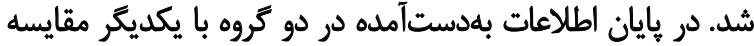

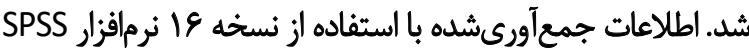
وآزمون تى تست تجزيه و تحليل شعد.

يأقتهها

تعداد •و بيمار مبتلا به سرطان خستان داراي معيارهاي ورود به

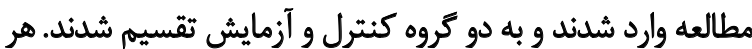

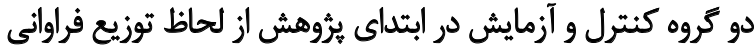

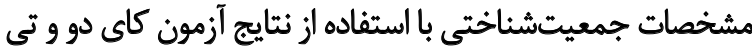

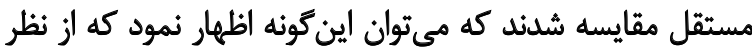

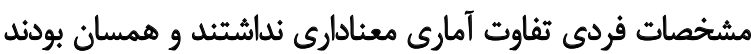

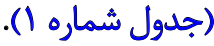

3. Western
ناخوشايند به تأخير بيندازند يا از ادامه درمان امتناع كنند و يا قادر به

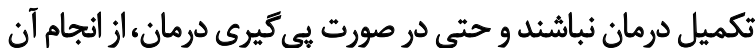

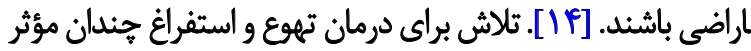

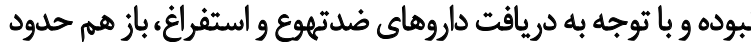

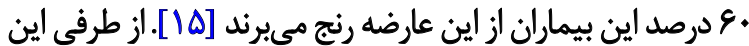

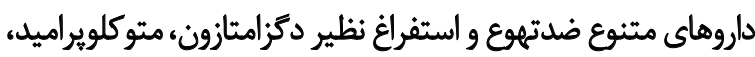

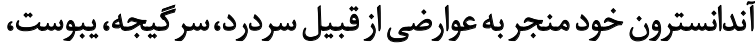

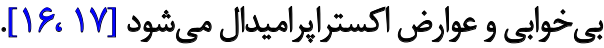
باتوجه به تأثير محدودو عوارض خطرناي (سئدرماكسترايراميدال،

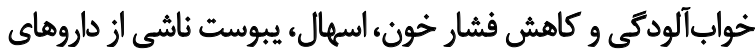

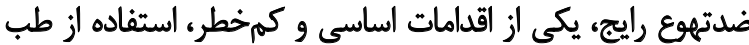

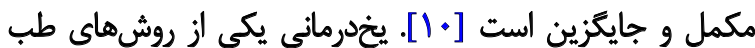

$$
\text { مكمل است. }
$$

يخدرمانى بر اين نظريه استوار است كه سرما با ايجاد انقباض

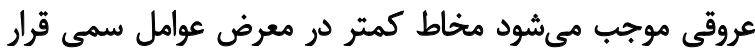

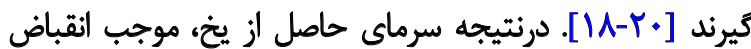
عروقى در قسمتهاى محيطى دستكاه كوارش (مرى و و معدها

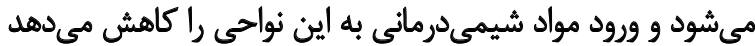

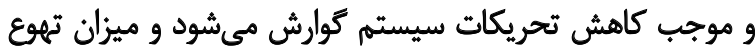

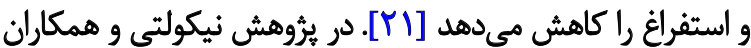

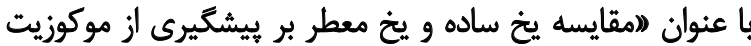

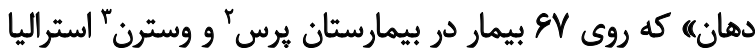

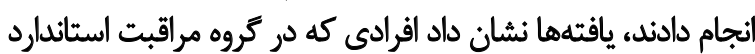

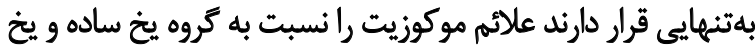
معطر بيشتر تجربه مي كنيند.

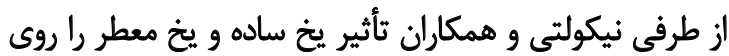

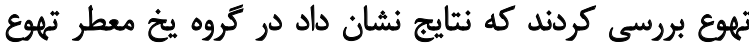

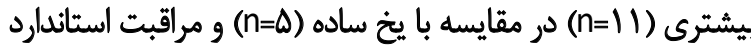

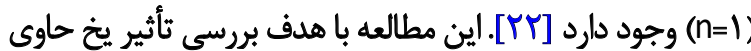

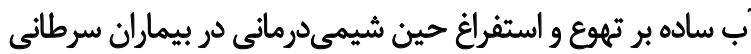
مراجعه كننده به بيمارستان اميد مشهد انجام شد. شئد

مواد و روشها

اين بُروهش از نوع كارآزمايى بالينى است. تعداد • • بيمار از ميان

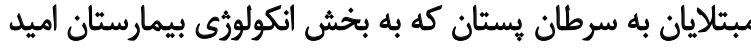

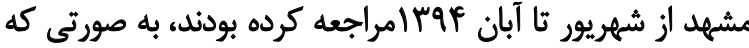

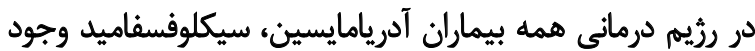

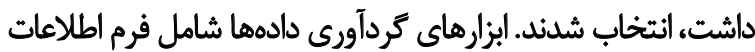

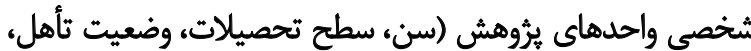

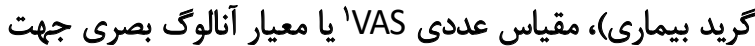

1. Visual Analogue Scale

2. Preth 
هدف بررسى تأثير ماساز يخ بر تهوع و استفراغ بيماران سرطانى

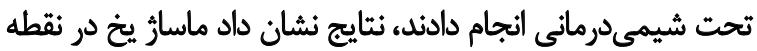

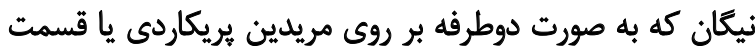

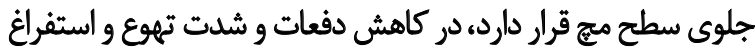

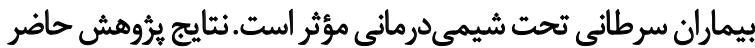

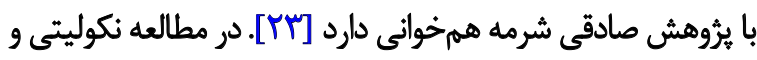

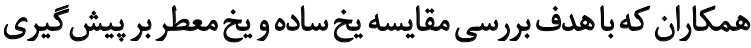

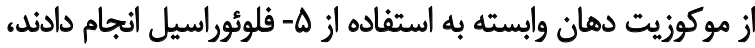

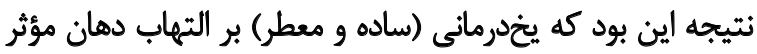

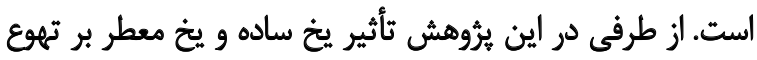

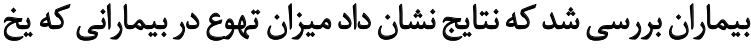

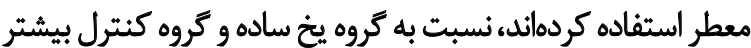

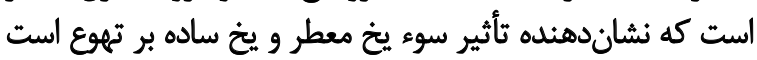

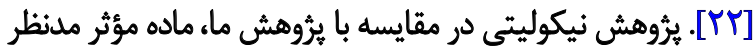

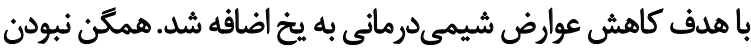

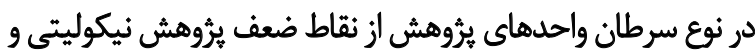
نقطه قوت آن همكن سازى بيماران از نظر رثيم شيمى درمانى است.

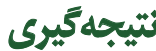

با توجه به نتايج مطالعه، استفاده از يخ حاوى آب ساده به عنوان

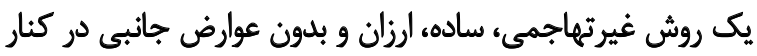
دارودرمانى در جهت بهبود تهرع بيماران سرطانى توصيه مي آشود.
همجينين در ابتداي يُروهش از نظر تهوع و استفراغ بررسى شدند و با

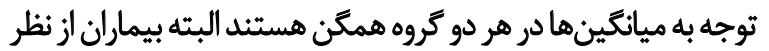

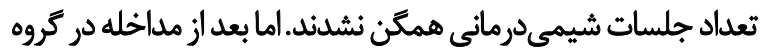

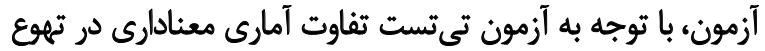

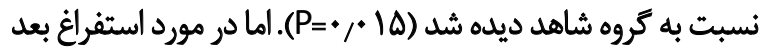

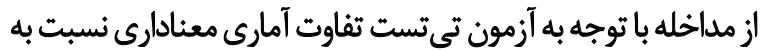

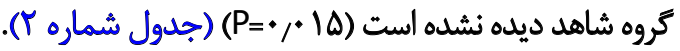
بحث

اين مطالعه با هدف بررسى تأثير مكيدن يخ حاوى آب ساده

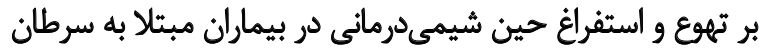

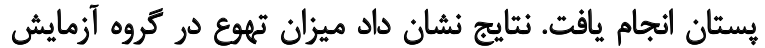

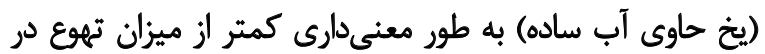

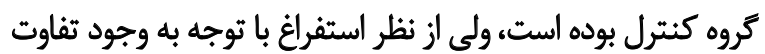

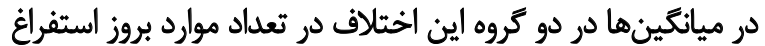

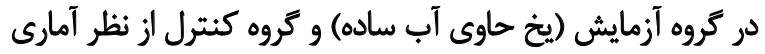

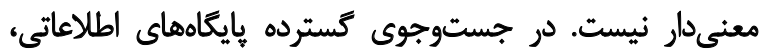

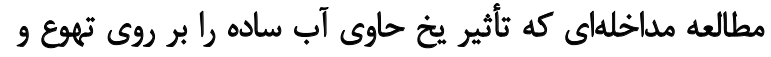

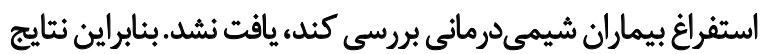

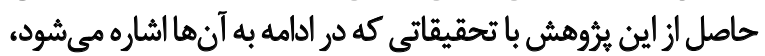

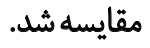

در مطالعهاي كه صادقى شرمه و همكاران در سال 1 11 با

جدول ا. وضعيث اطلاعات جمعيتشناختى در دو كروه مورد مطالعه (·r=r)

\begin{tabular}{|c|c|c|c|c|}
\hline \multirow{2}{*}{ نتيجه آزمون (P) } & \multicolumn{2}{|c|}{ تعداد (درصد) } & \multirow{2}{*}{ 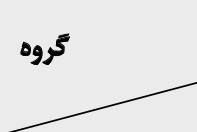 } & \multirow[b]{2}{*}{ 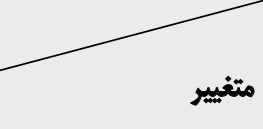 } \\
\hline & آزمايش & كنترل & & \\
\hline \multirow{4}{*}{$.18 .9^{\circ}$} & $1 \cdot(\pi / / T)$ & $V(\pi / / \pi)$ & خواندن وتوشتن & \multirow{4}{*}{ وضعيت تحصيلات } \\
\hline & $\mathbb{R}(\boldsymbol{r} \cdot)$ & $11(r / 99)$ & سيكل ل & \\
\hline & $\Delta(19199)$ & ᄉ (T\&/\&9) & دييلم & \\
\hline & $r(1)$. & $\mathbb{1 r}\left(1 \% / M^{e}\right)$ & ل ليساس & \\
\hline \multirow{2}{*}{.$(\mathrm{VN})^{*}$} & $r \cdot(\$ 9 / 9 V)$ & rT (Ne/9q) & مثاهل & \multirow{2}{*}{ وضعيت تأهل } \\
\hline & $1 \cdot(\pi / T \pi)$ & $V(1 r / \pi r)$ & مجرد & \\
\hline \multirow{4}{*}{. pere } & $11(\% / 99)$ & $g(r \cdot)$ & كريدا & \multirow{4}{*}{ كريد (درجه شدت) بيمارى } \\
\hline & $1 \cdot(\pi / / M)$ & $\pi(\% \pi / m)$ & كريدr & \\
\hline & $g(r \cdot)$ & $9(\mu \cdot)$ & كريدr & \\
\hline & $r(1 \cdot)$ & $r(\varepsilon / 9 V)$ & كريد & \\
\hline.$/ r^{*}$ & $\Lambda \pm \uparrow 1 / R \Delta$ & $r N V \pm V / M P$ & \multicolumn{2}{|c|}{ سن (سال) (ميانكين \#|نحراف معيار) } \\
\hline
\end{tabular}


جدول r. ميانكين و ائحراف معيار تهوع و استفراغ در دو كروه قبل و بعد از مداخله

\begin{tabular}{|c|c|c|c|}
\hline \multirow{2}{*}{ تتيجه آزمون(P)" } & \multicolumn{2}{|c|}{ ميانكين \#انحراف معيار } & \multirow{2}{*}{ تههع و استفراغ } \\
\hline & آزمايش & كتترل & \\
\hline - /AAF & $r+\varphi / f \pm 1 / 1$ & $\Delta T r / T A Y \pm I / I$ & تهوع قبل از مداخله \\
\hline .1 .10 & rENa土1/1 & Q/YAY $\pm 1 / r$ & تهوع بعد از هداخله \\
\hline . lsap & $\Pi \leftarrow \Delta / / \Pi \pm \cdot / \cdot$ & $r \cdot \Delta / \Delta \pm \bullet /$ & استفراغ قبل از مداخله \\
\hline . $/ \Delta F q$ & $F \cdot g / r \pm * /$ & RFq/YESDI/1 & استفراغ بعد از مداخله \\
\hline
\end{tabular}

\section{ملاحظات اخلاقى}

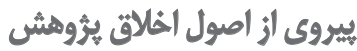

اين مقاله از طرح تحقيقاتى مصوب دانشكاه علوم يزشكى سبزوار با كد اخلاق شد. همجنين در مركز ثبت كار آرآمايى بالينى ايران با كد IRCT20160606028293N2

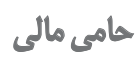

حامى مالى اين بروهش دانشكاه علوم يزشكى سبزوار است.

$$
\text { مشاركت نويسندكان }
$$

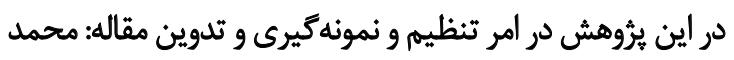

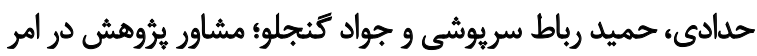

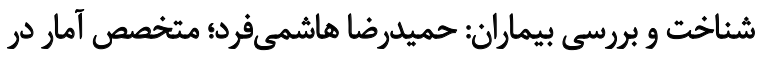
امر بررسى و انجام مسائل آمارى: ياسر تبرايى.

$$
\text { تمارض مثناقع }
$$

در اين مطالعه تضاد منافع وجود ندارد.

$$
\text { تشكر و قدردانى }
$$

برخود لازم مى دانيم از همكارى بيماران و يرسنل محترم بخش

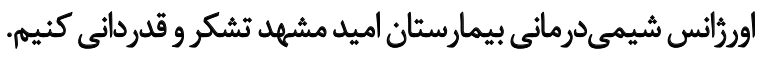

در اين يُووهش از يخ حاوى آب ساده استفاده شد. با توجه به اين

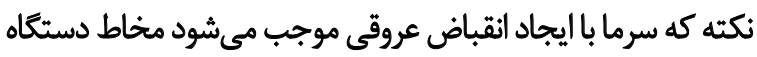

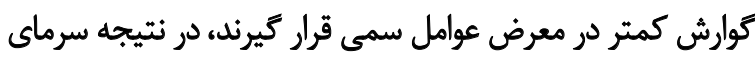

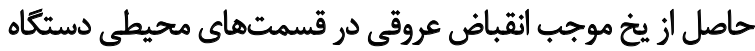

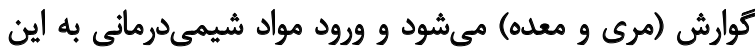

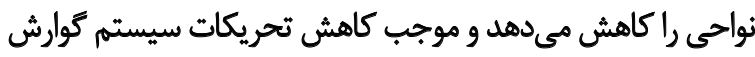

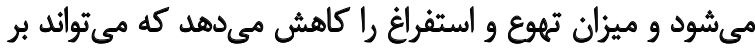

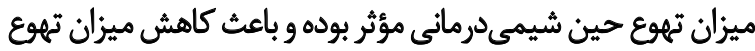

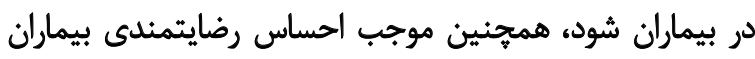

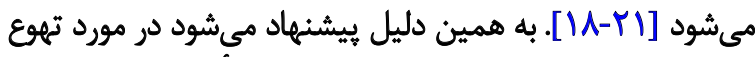

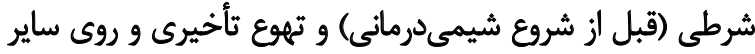
كروههاي سرطانى نيز استفاده شود.

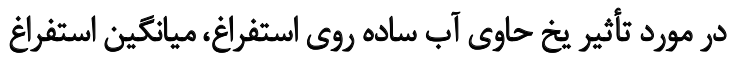

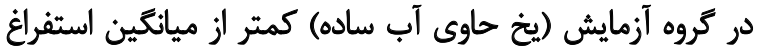

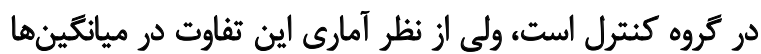

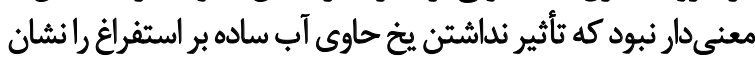

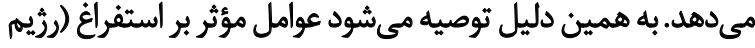
غذايى، اضطراب وغيره) بررسى شود.در نتيجه انجام مطالعات بيشتر

$$
\text { در اين زمينه توصيه مي إشود. }
$$

يافتههاى مطالعه در مورد استفراغ نشاندهنده تأثير نداشتن

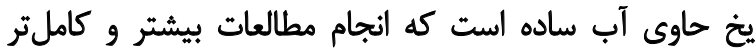

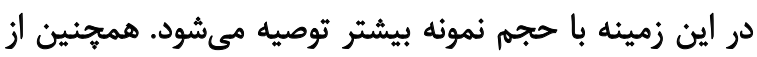

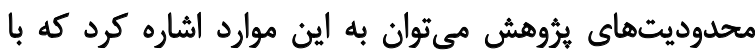

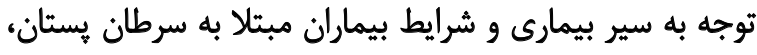

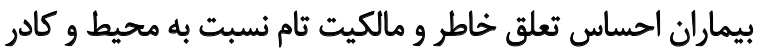

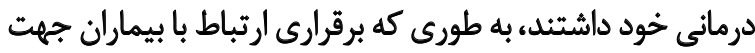

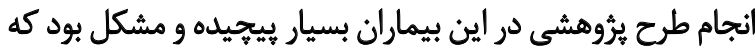

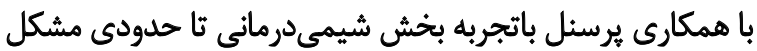

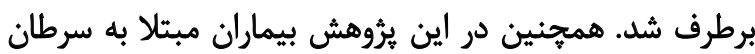

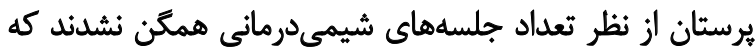

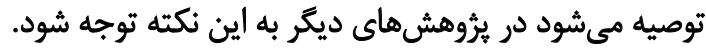




\section{References}

[1] Pudkasam S, Tangalakis K, Chinlumprasert N, Apostolopoulos V, Stojanovska L. Breast cancer and exercise: The role of adiposity and immune markers. Maturitas. 2017; 105:16-22. [DOI:10.1016/j.maturitas.2017.04.022] [PMID]

[2] Shinden Y, Kijima Y, Hirata M, Nakajo A, Tanoue K, Arigami T, et al. Clinical characteristics of breast cancer patients with mental disorders. The Breast. 2017; 36:39-43. [DOI:10.1016/j.breast.2017.08.010] [PMID]

[3] Mohammad Karimi M, Shariatnia K. [Effectiveness of spiritual therapy on the life quality of the women with breast cancer in Tehran (Persian)]. Journal of Urmia Nursing and Midwifery Faculty. 2017; 15(2):107-18.

[4] Bavadi B, Poursharifi H, Lotfi Kashani F. [The effectiveness of cognitive-behavioral therapy based 8-step pattern of cash to improve psychological well-being of women with breast cancer (Persian)]. Iranian Quarterly Journal of Breast Diseases. 2016; 8(4):41-51.

[5] Moghadamfar N, Amraei R, Asadi F, Amani O. [The efficacy of Acceptance and Commitment Therapy (ACT) on hope and psychological well-being in women with breast cancer under chemotherapy (Persian)]. Iranian Journal of Psychiatric Nursing. 2018; 6(5):1-7.

[6] Thomas E, Escandón S, Lamyian M, Ahmadi F, Setoode SM, Golkho Sh. Exploring Iranian women's perceptions regarding control and prevention of breast cancer. The Qualitative Report. 2011; 16(5):1214-29

[7] Mousavi SM, Gouya MM, Ramazani R, Davanlou M, Hajsadeghi N, Seddighi Z. Cancer incidence and mortality in Iran. Annals of Oncology. 2009; 20(3):556-63. [DOI:10.1093/annonc/mdn642] [PMID]

[8] Malekzade Moghani M, Peykani S, Amani O. [Comparative study of quality of life in women survived with those suffered from breast cancer while under chemotherapy (Persian)]. Journal of Health Promotion Management. 2017; 6(4):8-13. [DOI:10.21859/jhpm-07022]

[9] Chan A, Low XH, Yap K. Assessment of the relationship between adherence with antiemetic drug therapy and control of nausea and vomiting in breast cancer patients receiving anthracycline-based chemotherapy. Journal of Managed Care \& Specialty Pharmacy. 2012; 18(5):385-94. [DOI:10.18553/jmcp.2012.18.5.385] [PMID]

[10] Matory P, Gholamy R, Dehghan M, Vanaki Z, Shirazi M, Binaee N, et al. [Efficacy of complementary therapies in reduction of chemotherapy induced nausea and vomiting in breast cancer patients: Systematic review (Persian)]. Complementary Medicine Journal. 2014; $4(2): 831-44$.

[11] Arslan FT, Basbakkal Z, Kantar M. Quality of life and chemotherapyrelated symptoms of Turkish cancer children undergoing chemotherapy. Asian Pacific Journal of Cancer Prevention. 2013; 14:1761-8. [DOI:10.7314/APJCP.2013.14.3.1761] [PMID]

[12] Bourdeanu L, Frankel P, Yu W, Hendrix G, Pal S, Badr L, et al. Chemotherapy-induced nausea and vomiting in Asian women with breast cancer receiving anthracycline-based adjuvant chemotherapy. The Journal of Supportive Oncology. 2012; 10(4):149-54. [DOI:10.1016/j. suponc.2011.10.007] [PMID]

[13] Wiser W, Berger A. Practical management of chemotherapyinduced nausea and vomiting. Oncology (Williston Park). 2005; 19(5):637-45. [PMID]

[14] Matoury P, Vanaki Z, Zare Z, Mehrzad V, Dehghan M. [Investigation of the effects of therapeutic touch on intensity of anticipatory and acute induced-nausea in breast cancer women undergoing chemo- therapy in Isfahan (Persian)]. Complementary Medicine Journal. 2013; 3(3):585-94

[15] Marx W, Kiss N, McCarthy AL, McKavanagh D, Isenring L. Chemotherapy-induced nausea and vomiting: A narrative review to inform dietetics practice. Journal of the Academy of Nutrition and Dietetics. 2016; 116(5):819-27. [DOI:10.1016/j.jand.2015.10.020] [PMID]

[16] Rithirangsriroj K, Manchana T, Akkayagorn L. Efficacy of acupuncture in prevention of delayed chemotherapy induced nausea and vomiting in gynecologic cancer patients. Gynecologic Oncology. 2015; 136(1):82-6. [DOI:10.1016/j.ygyno.2014.10.025] [PMID]

[17] Eghbali M, Varaei Sh, Hosseini M, Yekaninejad MS, Shahi F. The effect of aromatherapy with peppermint essential oil on nausea and vomiting in the acute phase of chemotherapy in patients with breast cancer. Journal of Babol University of Medical Sciences. 2018; 20(9):66-71.

[18] Heydari A, Sharifi H, Salek R. Effect of oral cryotherapy on combination chemotherapy-induced oral mucositis: A randomized clinical trial. Middle East Journal of Cancer; 2012; 3(2\&3):55-64.

[19] Alvariño-Martín C, Sarrión-Pérez MG. Prevention and treatment of oral mucositis in patients receiving chemotherapy. Journal of Clinical and Experimental Dentistry. 2014; 6(1):e74-e80. [DOI:10.4317/ jced.51313] [PMID] [PMCID]

[20] Köstler WJ, Hejna M, Wenzel C, Zielinski CC. Oral mucositis complicating chemotherapy and/or radiotherapy: Options for prevention and treatment. A Cancer Journal for Clinicians. 2001; 51(5):290-315. [DOI:10.3322/canjclin.51.5.290] [PMID]

[21] Lane B, Cannella K, Bowen C, Copelan D, Nteff G, Barnes K, et al. Examination of the effectiveness of peppermint aromatherapy on nausea in women post C-section. Journal of Holistic Nursing. 2012; 30(2):90-104. [DOI:10.1177/0898010111423419] [PMID]

[22] Nikoletti S, Hyde S, Shaw T, Myers H, Kristjanson L. Comparison of plain ice and flavoured ice for preventing oral mucositis associated with the use of 5 fluorouracil. Journal of Clinical Nursing. 2005; 14(6):750-3. [DOI:10.1111/j.1365-2702.2005.01156.x] [PMID]

[23] Sadeghi Shermeh M, Ebadi A, Sirati Nir M, Azadian M. [Effect of ice massage on nausea and vomiting in patients under chemotherapy (Persian)]. Journal of Birjand University of Medical Sciences. 2012; 19(1):1-11 